\title{
Transcriptome analysis provides new insight into the molecular mechanisms in regulating carotenoid accumulation in pericarp of fruits from two pummelo cultivars Citrus grandis (L.) Osbeck
}

Xinkun Lu ( $\sim$ gsskg@126.com )

Institute of Pomology, Fujian Academy of Agricultural sciences https://orcid.org/0000-0003-1575-5919

Yanqing Lu

Institute of Pomology, Fujian Academy of Agricultural Sciecnes

Yanjin Lin

Institute of Pomology, Fujian Academy of Agricultural Sciences

Research article

Keywords: Citrus grandis (L.) Osbeck, Pericarp, RNA-seq, Carotenoid, Regulation

Posted Date: July 3rd, 2019

DOI: https://doi.org/10.21203/rs.2.10939/v1

License: (c) (i) This work is licensed under a Creative Commons Attribution 4.0 International License.

Read Full License 


\section{Abstract}

Background Carotenoid improves fruit external quality and benefits to human health. Although the mechanism underlining carotenoid metabolism is clear, the regulation of carotenoid accumulation remains poorly understand. Recently, 'Huangbao' pummelo was selected from the bud mutation of 'Guanxi' pummelo (yellow pericarp). The pericarp of mutant-type fruits is characterized by red colour development during fruit ripening stage. The aim of the study is to explore the molecular mechanisms in regulating pericarp colouration based on transcriptomic profile analysis of the two cultivars. Results Lycopene content in the pericarp of ripe fruits from 'Huangbao' (160.29 $\mu \mathrm{g} \mathrm{g}-1 \mathrm{FW})$ was significantly higher than that of from 'Guanxi' ( $0.91 \mu \mathrm{g} \mathrm{g}-1 \mathrm{FW})$. Lycopene is a main contributor for pericarp colouration. 929 differentially expressed genes (DEGs) were indentified through transcriptional profiling in the pericarp of ripe fruits from the two cultivars. Expression levels of the Genes encoding for fructosebisphosphate aldolase, enolase and pyruvate kinase isozyme were significantly higher in 'Huangbao' than that of in 'Guanxi', which possibly promote 3-phosphate-glyceraldehyde and pyruvic acid biosyntheses. This two productions act as precursors for the biosyntheses of Isopentenylpyrophosphate (IPP) and Dimethylallyl pyrophosphate (DMAPP) in Methylerythritol phosphate (MEP) pathway. Genes ecoding for phytoene synthase and prolycopene isomerase were unexpected down-regulated in 'Huangbao'. Gibberellin-2- $\beta$-dioxygenase (GA2OX) gene was specially expressed in 'Huangbao', while the transcript amounts of genes related to auxin response and auxin transport as well as several negative regulators in ethylene signaling in 'Huangbao' were all down regulated. Additionally, Transcription factors (DELLA, NAC, MADS-box, WRKY) and post translational modification proteins (histone acetyltransferase and E2 ubiquitin-conjugating enzyme) involved in regulating ethylene and carotenoid metabolisms were also differentially expressed in the two pummelo cultivars. Conclusions The main differences in the carotenoid metabolism in the pericarp of ripen fruits from 'Huangbao' and 'Guanxi' were observed in the carotenoid precursor biosynthetic pathway. Differentially expressed genes in gibberellin, auxin and ethylene metabolisms and plant hormone signaling pathways, as well as several transcription factors and post translational modification protein genes involved in regulating ethylene and carotenoid metabolisms provide targets for further exploration in revealing mechanisms underlying fruit colour development.

\section{Abstract}

Background: Carotenoid improves fruit external quality and benefits to human health. Although the mechanism underlining carotenoid metabolism is clear, the regulation of carotenoid accumulation remains poorly understand. Recently, 'Huangbao' pummelo was selected from the bud mutation of 'Guanxi' pummelo (yellow pericarp). The pericarp of mutant-type fruits is characterized by red colour development during fruit ripening stage. The aim of the study is to explore the molecular mechanisms in regulating pericarp colouration based on transcriptomic profile analysis of the two cultivars.

Results: Lycopene content in the pericarp of ripe fruits from 'Huangbao' (160.29 $\left.\mathrm{g} \mathrm{g} \mathrm{g}^{-1} \mathrm{FW}\right)$ was significantly higher than that of from 'Guanxi' ( $\left.0.91 \mu \mathrm{g} \mathrm{g}^{-1} \mathrm{FW}\right)$. Lycopene is a main contributor for 
pericarp colouration. 929 differentially expressed genes (DEGs) were indentified through transcriptional profiling in the pericarp of ripe fruits from the two cultivars. Expression levels of the Genes encoding for fructose-bisphosphate aldolase, enolase and pyruvate kinase isozyme were significantly higher in 'Huangbao' than that of in 'Guanxi', which possibly promote 3-phosphate-glyceraldehyde and pyruvic acid biosyntheses. This two productions act as precursors for the biosyntheses of Isopentenylpyrophosphate (IPP) and Dimethylallyl pyrophosphate (DMAPP) in Methylerythritol phosphate (MEP) pathway. Genes ecoding for phytoene synthase and prolycopene isomerase were unexpected down-regulated in 'Huangbao'. Gibberellin-2- $\beta$-dioxygenase (GA2OX) gene was specially expressed in 'Huangbao', while the transcript amounts of genes related to auxin response and auxin transport as well as several negative regulators in ethylene signaling in 'Huangbao' were all down regulated. Additionally, Transcription factors (DELLA, NAC, MADS-box, WRKY) and post translational modification proteins (histone acetyltransferase and E2 ubiquitin-conjugating enzyme) involved in regulating ethylene and carotenoid metabolisms were also differentially expressed in the two pummelo cultivars.

Conclusions: The main differences in the carotenoid metabolism in the pericarp of ripen fruits from 'Huangbao' and 'Guanxi' were observed in the carotenoid precursor biosynthetic pathway. Differentially expressed genes in gibberellin, auxin and ethylene metabolisms and plant hormone signaling pathways, as well as several transcription factors and post translational modification protein genes involved in regulating ethylene and carotenoid metabolisms provide targets for further exploration in revealing mechanisms underlying fruit colour development.

Keywords: Citrus grandis (L.) Osbeck, Pericarp, RNA-seq, Carotenoid, Regulation

\section{Background}

It was found that Carotenoids are involved in developmental process in organisms, and specially synthesized in plants and microorganisms. As one kind of light harvesting pigments, Carotenoids impact the photosynthetic activity in plants $[1,2]$. Carotenoids elevate oxidation resistance in plants due to their roles in scavenging active oxygen species, and preventing their biosynthesis [3,4]. Carotenoids affect phytohormone biosynthesis, for examples, Isopentenylpyrophosphate (IPP) is the common precursor for carotenoid and gibberenllin (GA) biosyntheses [5]; the intermediate product in the downstream metabolic pathway of carotenoids is the precursor for abscisic acid biosynthesis [6, 7]. Carotenoid composition determines plant tissue colours, and more important, carotenoid components are beneficial for hunman health [8]. Due to the fact that carotenoids participating in metabolic processes in plants and humans, much extensive attention is concentrated on exploring the knowledge of carotenoid metabolism.

The whole process of carotenoid biosynthesis can be devided into two periods: the previous and following carotenoid precursor biosynthesis. IPP and Dimethylallyl pyrophosphate (DMAPP) are synthesized through mevalonic acid and methylerythritol phosphate (MEP) pathways, and then act as precursors for carotenoid biosynthesis. MVA and MEP pathways exist in the cytosol and the plastid of plants, respectively. Carotenoids are synthesized mainly through MEP pathway in plants [5]. IPP and 
DMAPP are catalyzed by Geranylgeranyl pyrophosphate synthase to form geranylgeranyl diphosphate (GGPP). Phytoene synthase (PSY) catalyzes condensation of two molecules of GGPP, forming the phytoene. Phytoene is desaturated by phytoene desaturase (PDS) to yield $\zeta$-carotene, and then the desaturation of $\zeta$-carotene is catalyzed by $\zeta$-carotene desaturase (ZDS) to produce tetra-cis-lycopene. Carotenoid isomerase (CRTISO) converts tetra-cis-lycopene to all-trans-lycopene. Lycopene $\beta$-cyclase ( $\beta$ LCY) catalyzes the cyclation of all-trans-lycopene to form $\beta$-carotene, however, when the cyclation of alltrans-lycopene is catalyzed by $\beta$-LCY and lycopene $\varepsilon$-cyclase $(\varepsilon$-LCY), $a$-carotene is formed. $\beta$-ring carotene hydroxylase $(\mathrm{BCH})$ and $\varepsilon$-ring carotene hydroxylase $(\mathrm{E} \mathrm{CH})$ catalyze the hydroxylation of $\alpha-$ carotene, yielding the lutein. $\beta$-cartoene is hydroxylated only by $\mathrm{BCH}$, converting to zeaxanthin.

Zeaxanthin epoxidase converts zeaxanthin to antheraxanthin and violaxanthin. Violaxanthin is converted to neoxanthin by neoxanthin synthase at the last step [9].

The modulation of carotenoid accumulation is not limited in the carotenoid metabolic pathway. The carotenoid distribution in cell organelles also impacts carotenoid levels in plants. Chromoplasts act as the sink for carotenoids, their structure changes parallel carotenoid accumulation. Most chromoplasts in mature green fruits of red-fleshed papaya (Carica papaya L. cv. Pococí) were characterized by widely undifferentiated stroma, then the plastoglobules could be observed in chromoplasts during fruit development, at ripening stage, crystal-type lycopene formed in numerous chromoplasts, meanwhile, plastoglobules and tubular structures could still be indentified [10]. The final structural characteristics of chromoplasts are correlated with carotenoid composition. For example, lycopene accumulation in pulp of fruits from red-fleshed 'Cara Cara' (Citrus sinensis L. Osbeck) and Star Ruby (Citrus paradise Macf.) was linked to the formation of needle-like crystal in chromoplasts, $\beta, \beta$-xanthophylls accumulation in 'Navel orange' (Citrus sinensis L. Osbeck) fruit pulp led to the formation of plastoglobule in chromoplasts, however, the elevated colourless carotene contents in 'Pinalate' (Citrus sinensis L. Osbeck) fruit pulp were associated with the development of numerous vesicles in chromoplasts [11]. The component analysis of chromoplasts in tomato (Solanum lycopersicum) fruits showed that the increased plastid-localized lipid monogalactodiacylglycerol content in chromoplast membrane, was correlated with the promoted capacity for $\beta$-carotene and lycopene in chromoplasts [12]. These reports indicate that the concurrent changes in chromoplast structures with carotenoids may offer feedback information for carotenoid biosynthetic metabolism in plants. Some other metabolic pathways are capable of impacting carbon skeleton supply for carotenoid biosynthesis or controlling carotenoid degradation, consequently, influence carotenoid accumulation. [7]. Environmental factors also play important roles in regulating carotenoid metabolism. Light quality and illumination time regulate carotenoid contents and transcript levels of the genes involved in carotenoid biosynthesis and degradation [13, 14]. In an addition, soil [15] and mineral elements [16-18] also exhibite effects in modulating carotenoid contents in plants. The fluctuations of carotentoid contents in plants cultivated in different geographical conditions may be caused by the influences of external environmental factors at least in part [19]. Even the carotenoid biosynthesis in fruits during storage is also influenced by environment. The previous reports have been showed that high storage temperature $\left(12-20^{\circ} \mathrm{C}\right)$ conditioning significantly promoted carotenoid biosynthsis in fruits when compared to low storage temperature $\left(4^{\circ} \mathrm{C}\right)[20,21]$. Therefore, the approaches in regulation of 
carotenoid accumulation are not limited in the core carotenoid metabolic pathway, which can be extend to the carotenoid precursor metabolic pathway and the carotenoid distribution in plastids. The above states can partially explain why the transcript levels of genes involved in core carotenoid metabolism are not always coincident with carotenoid accumulation [22, 23].

Carotenoid accumulation is directly associated with plant genotype. $\beta$-cryptoxanthin content in the pericarp of mandarin (Citrus reticulata Blanco) fruits was significantly higher than that in the pericarp of fruits from sweet orange (Citrus sinensis (L.) Osbeck) (10-fold), lemon (Citrus limon L.) (15-fold) and pummelo (Citrus grandis (L.) Obseck) (200-fold) [24]. Distinct differences in carotenoid contents even could be find in different sweet orange cultivars, for instance, $\zeta$-carotene and lutein mainly accumulated in 'Maltaise demi-sanguine' fruits, however, lycopene was the main contributor for carotenoid accumulation in 'Maltaise demi-sanguine' fruits [25]. The same trend was observed in vegetables [26, 27] and flowers [28].

it is necessary to carry out experiments using plant materials with different colour development to explore the mechanisms in regulating carotenoid accumulation, due to the fact that both of genetic background and environmental conditions influence carotenoid metabolism. 'Huangbao' pummelo was derived from the bud mutation of 'Guanxi' pummelo. The pericarp of the mutant-type fruits is characterized by the red colour development during fruit ripening stage, while the wild-type pericarp still keeps yellow even at the fully ripe stage. The aim of the present study is to explore molecular metabolisms in regulating carotenoid accumulation based on transcriptome analysis in the pericarp of ripe fruits from 'Huangbao' and 'Guanxi' pummelo.

\section{Results}

RAN-sequencing and assembly of unigenes

Six cDNA libraries were created using 'Huangbao' and 'Guanxi' samples (i.e. each cultivar with three independent biological replicates). Raw reads were generated from the Illumina sequencing, and clean reads were obtained by filtering the adaptor sequences and the low quality reads. The average GC content of clean reads was nearly the same (45\%), and the average Q30 base percentage was more than $92 \%$. Those high quality reads were de novo assembled using trinity method, generating 97377 unigenes with an mean length of $730 \mathrm{nt}$, and a N50 length of $1188 \mathrm{nt}$. The size distribution of unigenes was showed in Fig. 1. More than $78 \%$ of the clean reads per sample could mapped on to the assemlbled unigenes (Table 1 in Supplementary Files).

Functional annotation of unigenes

A total of $55820(57.32 \%)$ all unigenes and $826(88.91 \%)$ differentially expressed unigenes (DEGs) were annotated based on sequence alignment in 8 public databases, including NCBI non-redundant protein sequences (NR), Protein family (Pfam), a manually annotated and reviewed protein sequence database (Swiss-Prot), gene ontology (GO), Clusters of Orthologous Groups (COG), euKaryotic Orthologous Groups 
(KOG), Evolutionary Genealogy of Genes: non-supervised orthologous groups (eggNOG) and Kyoto Encyclopedia of Genes and Genomes (KEGG) (Table 2 in Supplementary Files).

The unigenes were blasted against the NR database with a cut-off e-value of $<1 e^{-5} .54 .88 \%$ of the annotated unigenes had significant homology matches (e-value $<1 \mathrm{e}^{-50}$ ) in this database (Fig. 2a). Species specific distribution showed that $27.20 \%$ of the unigenes matched gene sequences of Citrus sinensis, followed by Citrus clementina (15.04\%), Elaeis guineensis (7.27\%), Phoenix dactylifera (6.45\%), Musa acuminate (3.21\%), and other species (30.81\%) (Fig. 2b).

All unigenes were annotated within $\mathrm{GO}$ database into three main categories, including biological process (BP) (20 terms), molecular founction (MF) (17 terms) and cellular component (CC) (19 terms) (Fig. 3). In the BP category, the main enriched GO terms included metabolic process, cellular process, response to stimulus, biological regulation, localization and signaling, which indicated that the metabolic activities still maintained high levels in the pericarp even at fruit ripening stage. In case of MF, most of the genes belonged to catalytic activity, binding, transporter activity, nucleic acid binding transcription factor activity, enzyme regulator activity, and protein binding transcription factor activity, which suggested that those genes possibly involved in regulation of metabolisms. As far as CC, the predominant subcategories were cell part, organelle, membrane, macromolecular complex, membrane part, membrane-enclosed lumen, cell junction and symplast.

Functional annotation of unigenes in COG, KOG and eggNOG databases indicated that large number of unigenes were calssificated into several functional categories, including general function prediction only, posttranslational modification, carbohydrate transport and metabolism, signal transduction mechanisms, translation and transcription (Additional file 1). It was also found that large number of unigenes were encriched in carbon metabolism and ribosome pathways based on the KEGG pathway enrichment analysis (Additional file 2).

Global detection of DEGs

The comparison of transcriptomes of the two pummelo cultivars identified 929 DEGs showing a significant change in expression level (FDR $<0.01$ and fold change $\geq 2$ ), including 326 up-regulated genes and 603 down-regulated genes (additional file 3). The fold changes of the majority of those DEGs were distributed in the range of 2-10. It was investigated that 130 and 150 DEGs specially expressed in the pericarp of ripe fruits from 'Huangbao' and 'Guanxi', respectively. $90 \%$ of those specially expressed DEGs exhibited a low transcript level $(F P K M<5)$. Cluster analysis of the total DEGs based on log2(FPKM) was provided in Fig. 4.

GO enrichment analysis of DEGs

GO enrichment analysis was performed using a threshold value of $p \leq 0.05$. In the group of up-regulated genes, there were 42, 14 and 37 subcategories were significantly enriched in BP, CC and MF, respectively, while in the down-regulated gene group, there were 55,13 and 58 subcategories were significantly 
enriched in BP, CC, and MF, respectively. TopGO analysis showed that cell surface receptor signaling pathway (GO: 0007166) is one of the most significantly enriched subcategories in the BP category. In the case of CC, plastid (GO: 0009536), chloroplast (GO: 0009507), plastid part (GO: 0044435) and chloroplast part (GO: 0044434) were the most significantly enriched subcategories. As far as the MF, catalytic activity (GO: 0003824) and DNA binding (GO: 0003677) were the highest represented subcategories. The top ten classes from each categories were displayed in Additional files 4-6.

In addition to the above results, other significantly enriched subcategories were also obtained. In BP category, almost all of the genes calssified into translation (GO: 0006412) and isoprene biosynthetic process (GO: 0043612) were specially identified in the pericarp of ripe fruits from 'Huangbao'. Other relevant over-represented subcategories included: light harvesting (GO: 0009765), metabolic process (GO: 0008152), oxidation-reduction process (GO: 0055114), auxin transport (GO: 0010540) and transmembrane receptor protein serine/threonine kinase signaling pathway (G0: 0007178).

In terms of $\mathrm{CC}$, genes classified into the significantly enriched subcategories such like ribosome (GO: 0005840), plastoglobuli (GO: 0010287) and cytosolic small ribosomal subunit (GO: 0022627) were almost only detected in the pericarp of ripe fruits from 'Huangbao'. Other significantly enriched subcategories such like anchored component of plasma membrane (GO: 0046658), extrinsic component of membrane (GO: 0019898), integral component of membrane (GO: 0016021), cytoplasm (GO: 0005737) and vacuole (GO: 0005773) were also observed in CC.

As far as the MF category, most of the genes categorized into structural constituent of ribosome (GO: 0003735) and ribulose-1,5-bisphosphate carboxylase/oxygenase activator activity (GO: 0046863) only expressed in the pericarp of ripe fruits from 'Huangbao'. Monooxygenase activity (GO: 0004497) and potassium ion binding (GO: 0030955), carotenoid isomerase activity (GO: 0046608), metal ion binding (GO: 0046872) and hydroxymethylglutaryl-CoA reductase activity (GO: 0042282) were also the significantly enriched groups.

KEGG pathway enrichment analysis of DEGs

A sum of 211 out of 929 DEGs were enriched in 89 KEGG pathways, among which, ten pathways were significantly encriched $(P<0.05)$ (Additional file 2). Ribosome (ko03010) contained a high number of DEGs, in which, most of those genes encoding for $60 \mathrm{~S}$ ribosomal proteins and $40 \mathrm{~S}$ ribosomal proteins and specially expressed in the pericarp of ripe fruits from 'Huangbao' pummelo. Followed by the carbon fixation in photosynthetic organisms pathway (ko00710), genes enriched in this pathway were mainly annotated as glyceraldehyde-3-phosphate dehydrogenase, fructose-bisphosphate aldolase, and ribulose bisphosphate carboxylase small chain. Other unsignificantly enriched pathways contained few genes, such like arachidonic acid metabolism (ko00590), terpenoid backbone biosynthesis (ko00900), sesquiterpenoid and triterpenoid biosynthesis (k000909), carotenoid biosynthesis (ko00906), plant hormone signal transduction (ko04075), pyruvate metabolism (ko006200) and ubiquitin mediated proteolysis (ko04120). 
DEGs involved in carotenoid and its precursor metabolisms

Phytoene synthase and prolycopene isomerase are two enzymes that catalyze chemical reactions previous to the lycopene biosynthetic step. In this study, expression levels of genes ecoding for the two enzymes were significantly lower in the pericarp of ripe fruits from 'Huangbao' compared with 'Guanxi'.

Glyceraldehyde-3-phosphate and pyruvate are the precursors for IPP and DMAPP. We indentified an upregualted gene (c79299.graph_c0) encoding for fructose-bisphosphate aldolase, and two genes (c58950.graph_c0, c49982.graph_c0) encoding for an isozyme of fructose-bisphosphate aldolase. c58950.graph_c0 and c49982.graph_c0 were specially expressed in 'Huangbao' and 'Guanxi', respectively. Fructose-bisphosphate aldolase catalyzes the formation of glyceraldehyde-3-phosphate. An up-regulated gene (c50907.graph_c0) was annotated as enolase and specially expressed in 'Huangbao'. Enolase catalyzes the conversion of 2-diphosphoglycerate to Phosphoenolpyruvate. Three genes (c58669.graph_c0, c70874.graph_c0, c55863.graph_c0) were indentified for pyruvate kinase isozyme and highly expressed in 'Huangbao' when compared to 'Guanxi'. Pyruvate kinase catalyzes the conversion of phosphoenolpyruvate to pyruvate.

Three genes (c36907.graph_c0, c54686.graph_c0, c38780.graph_c0) involved in MVA pathway were down regualted in 'Huangbao' and annotated as Acetyl-CoA acetyltransferase, Hydroxymethylglutaryl-CoA synthase and 3-hydroxy-3-methylglutaryl-coenzyme A reductase, respectively. Transcript levels of the three genes indicated that MVA pathway activity in 'Huangbao' was lower than that in 'Guanxi'. The above genes were listed in Table 3 (in Supplementary Files.

DEGs involved in Other metabolic pathways

The DEGs analyses showed that all of the genes involved in biosynthesis and degradation of fatty acid were down regulated in 'Huangbao'. In contrast, most of the genes annotated as 40S and 60S ribosomal proteins were up regulated in 'Huangbao'. In addition, all of the genes encoding for elongation factor and cytochrome c oxidase subunit were also up regulated. Those genes exhibited distinct differences in transcript levels were listed in Additional file 7.

DEGs participated in phytohormone metabolic pathways

In the present study, several genes involved in gibberellin, cytokinin and ethylene metabolic pathways were differentially expressed between 'Huangbao' and 'Guanxi' cultivars. Genes encoding for Gibberellin 2- $\beta$-dioxygenase (GA2OX) and glycine-rich RNA-binding protein (GRP) were specially indentified in 'Huangbao' and 'Guanxi', respectively. Expression level of cytokinin dehydrogenase gene was down regulated in 'Huangbao'. A gene annotated as 1-aminocyclopropane-1-carboxylate synthase (ACS) was down-regulated in 'Huangbao', however, transcript level of one gene econding for 1-aminocyclopropane-1carboxylate oxidase (ACO) elevated 25.69 folds in 'Huangbao' compared with 'Guanxi' (Table 4 in Supplementary Files).

DEGs associated with signal transduction 
DEGs related to auxin and ethylene responsive proteins were down regulated in 'Huangbao'. Four genes were identified for auxin responsive proteins, one of them was annotated as putative indole-3-acetic acidamido synthetase $(\mathrm{GH} 3)$. In plants, this protein plays an important role in balancing active and inactive auxin contents through by catalyzing the synthesis of indole-3-acetic acid-amido. Two genes (c67065.graph_c0b, c69145.graph_c0) were not identified in 'Huangbao', one of which (c67065.graph_c0b) was annotated as EIN3-binding F-box protein 1 (EBF1), a negative factor in ethylene signaling pathway, while the other one encoded ethylene insensitive 3 (EIN3), a positive factor in ethylene signal transduction (Table 5 in Supplementary Files).

Many DEGs were found to be associated with other signaling pathways. Those genes were mainly annotated as calmodulin, tyrosine kinase, leucine-rich repeat receptor-like protein kinase, cysteine-rich receptor-like protein kinase, serine/threonine-protein kinase, histidine kinase, rapid alkalinization factor, protein phosphatase and non-specific lipid-transfer protein. It was observed that a largest number of genes were identified for serine/threonine-protein kinase. Most of these genes involved in other signaling pathways were down regulated in 'Huangbao' (Table 6).

Differentially expressed transcription factors

Some transcription factors related to fruit ripening process, phytohormone signal transduction, pigment metabolism and terpene biosynthesis, such like ethylene response transcription factor, MYB, DELLA, MADS-box and WRKY, were down regulated in 'Huangbao'. However, most of the NAC domain-containing protein genes showed up regulation (Table 7 in Supplementary Files).

DEGs participated in post-translational modification

Transcript levels of genes encoding for histone acetyltransferase and monothiol glutaredoxin were up regulated in 'Huangbao', in contrast, 14-3-3-like protein gene showed down regualtion. It was found that two genes were annotated as ubiquitin-conjugating enzyme E2, the highly expressed one was up regulated in 'Huangbao'. The two genes encoding for glutathione S-transferase showed the same trend (Table 8 in Supplementary Files).

DEGs related to substance transport

Nine genes encoding for the members of ATP binding cassette (ABC) family, except one up regulated gene, the rest of them were down regulated in 'Huangbao'. Gene expression of ion transporter in 'Huangbao' showed down regulation. Water and sugar transporter genes were up or down regulated in 'Huangbao' (Tables 9-12 in Supplementary Files).

Mesurement of carotenoid content

Carotenoid levels in the pericarp of ripe fruits from 'Huangbao' and 'Guanxi' were identified using high performance liquid chromatography (HPLC). In 'Huangbao' pummelo, lycopene was the main contributor for carotenoid accumulation, lutein and zeaxanthin were not indentified. However, minor amounts of 
carotenoids were detected in 'Guanxi' pummelo (Table 13 in Supplementary Files). HPLC chromatograms and standard curves were displayed in Additional file 8.

Validation of DEGs by quantitative real time PCR (qRT-PCR)

Eight genes were randomly selected and used to verify the stabilization of the transcriptome analysis results. qRT-PCR assay revealed that the relative expressions of the tested genes were coincident with the transcriptome profiles (Fig. 5). Primer and gene sequneces were listed in Additional file 9.

\section{Discussion}

Carotenoid precursor metabolisms influence carotenoid accumulation

Glyceraldehyde-3-phosphate and pyruvate are the precurcors for IPP and DMAPP synthesized through MEP pathway [5]. Fructose-1,6-phosphate conversion to glyceraldehyde-3-phosphate is catalyzed by fructose-bisphosphate aldolase. Enolase catalyzes the formation of phosphoenolpyruvate, and then, pyruvate kinase converts phosphoenolpyruvate to pyruvate. The expression levels of those genes encoding for the three enzymes were significantly higher in 'Huangbao' than that in 'Guanxi'. Those results indicated that the biosyntheses of the original precursors for carotenoids may be promoted in red pericarp of ripe fruits from 'Huangbao'.

The transcript levels of the genes annotated as PSY and CRTISO were distinctly lower in 'Huangbao' than that of in 'Guanxi'. It was investigated that the transcript abundance of PSY was not always coincident with the lycopene accumulation in tomato cultivar, its expression level at fruit ripening stage decreased 4 folds when compared with the colour break stage [26]. It has been reported that amino acid sequences influenced the activities of PSY and CRTISO [29-31], which suggests that translation regulation should not be ignore. It has been found that down regulation of $\beta-L C Y$ and capsanthin/capsorubin synthase gene involved in the downstream of carotenoid biosynthetic pathway contributed to lycopene accumulation in the pulp of 'Hong Anliu' sweet orange fruits [32]. Taken together, it seemed that expression levels of genes encoding for PSY and CRTISO had no direct impact on lycopene accumulation in citrus fruits.

Candidate genes involved in regulating carotenoid metabolism

Genes participated in phytohormone metabolisms and hormone signaling pathway

Carotenoid accumulation in tomao fruits during ripening stage was accompanied by the decreases of GA contents [33]. The application of exogenous GA led to increases in chlorophyll contents and decreases in carotenoid levels in the pericarp of lemon fruits [34]. GA are not likely to facilitate carotenoid accumulation in fruits. In this study, GA2OX and GRP were specially expressed in 'Huangbao' and 'Guanxi', respectively. Active GA (GA1 and GA4) were decreased by GA2OX reaction [35]. Overexpression of GRP caused decrease in transcript abundance of a gene encoding for the first-step enzyme in GA biosynthetic pathway, accordingly, the contents of GA4 and intermediate products of GA decreased [36]. 
The results of this study showed that the genes involved in regulating active GA contents were different between the two pummelo cultivars.

Exogenous cytokinin was capable of increasing both contents of chlorophyll and carotenoids [37-39]. Inhibition of expression of cytokinin dehydrogenase (CKX) gene was accompanied by the increase in endogenous cytokinin content [40]. A gene annotated as CKX was down regulated in 'Huangbao' pummelo. This result indicated that the rate of cytokinin degradation in the pericarp of 'Huangbao' ripe fruits might be lower when compared to 'Guanxi' and consequently improve carotenoid accumulation in part.

Ethylene palys a key role in regulating fruit ripening process. It has been observed that improvement of nutritional quality and colour development in fruits were influenced by ethylene [41]. Less ethylene production led to less carotenoid content in tomato fruits, correspondently, the orange fruit could not turn into red even at ripening stage [42]. The application of 1-methylcyclopropene (an inhibitor for ethylene biosynthesis) delayed colour development in citrus fruits [43]. By contrast, exogenous ethylene application elevated carotenoid contents in loquat fruits and accelerated fruit colour change [44]. ACO catalyzes the final step of ethylene biosynthetic pathway. ACO3 was down regulated by virus induced gene silencing in hot pepper (Capsicum frutescens) fruits, resulting in inhibition of fruit colouration, which reveals the relationship between ethylene metabolism and fruit colour development [45]. ACS catalyzes the formation of 1-aminocyclopropane-1-carboxylate (ACC) [46]. ACO catalyzes the conversion of ACC to ethylene, and acts as a positive regulator in ethylene biosynthesis [47]. In the present study, although the expression level of ACS was lower in 'Huangbao' than that of in 'Guanxi', $A C O$ was highly transcripted in 'Huangbao', the FPKM value was up to 1458.83 . Those results indicated that ethylene is synthesized in the pericarp of fruits from both of the two cultivars. Ten DEGs were identified in ethylene signaling pathway, one of them was $E B F 1$, and the rest of them were enthylene responsive transcription factors, including members in ERF and RAP2 subfamilies and EIN3. They were all lowly expressed in 'Huangbao'when compared with 'Guanxi', in particular, EBF1 and EIN3 were only detected in 'Guanxi'. ERF and RAP2 subfamilies belong to the family of ethylene responsive element binding protein and contain many members. For example, a total of 102 ERFs and 18 AP2s were identified in 'Newhall' sweet orange [48]. In tomato, RNAi repression of SIERF6 resulted in increases in carotenoid and ethylene contents [49]. The RNAi approach was also used to reduce SIAP2a expression level, the results showed that ethylene was over producted but carotenoid levels were decreased in tomato fruits [50]. Those reports indicate that ERF and AP2 both are negative regulators in ethylene signaling pathway, but play opposite roles in modulating carotenoid accumulation in fruits. EIN3 is a critical transcription factor in ethylene signal transduction pathway [51]. In addition, it is also involved in regulation of carotenoid metabolism. In papaya fruits, elevated transcript abundance of CpEIN3a accompanied with increased carotenoid content and up regulated genes in carotenoid biosynthetic pathway (CpPDS2/4, CpZDS, CpLCY-e and CpCHY-b) [52]. SIEIN2 silenced tomato fruits could not turn into red at ripening stage due to the inhibition in lycopene biosynthesis [53]. EBF expression is influenced by feedback loop of ethylene signaling, and then involved in fine-tuning of ethylene signal transduction. EIN3 transcription factor could interact with EBF2 promoter and directly elelvated its expression level [51]. EBF belongs to F-box protein family and acts as a 
component in SCF complex, this complex could degrade EIN3 protein [54]. Recently, it has been reported that SIEBF3 could interact with all known ethylene insensitive-like proteins (EIL) in tomato fruits and overexpression of SIEBF3 reduced the total SIEIL protein levels [55]. Consequently, EBF protein influences the sensitiveness of plant to ethylene. Taken together, the results obtained in the present study suggested that the high expression levels of negative transcription factors in ethylene signal pathway were capable of inhibiting ethylene signaling, finally, at least in part, led to carotenoids were weakly synthesized in the pericarp of ripe fruits from 'Guanxi'.

Application of indole-3-acetic acid (IAA) decreased transcript levels of several genes (PSY1, PSY3, PDS, $Z I S O$ and $C R T I S O$ ) involved in the up-stream carotenoid metabolic pathway, but induced expression of down-stream genes ( $\beta$-LCY1 and CRTR- $\beta 1$ ). Consequently, tomato fruit could not turn into red due to the significant decrease in lycopene content and remarkable increase in violaxanthin and neoxanthin contents [56]. GH3 playes an important role in auxin homeostasis by converting active auxin into inactive IAA-amino acid conjugate [57]. A sum of 24 members in $G H 3$ family were indentified in tomato fruits, in SIGH3-2 silenced fruits, the peak of ethylene production was delayed, finally, lycopene content decreased remarkably [58]. It can be observed that $\mathrm{GH} 3$ influences fruit carotenoid content through by modulating auxin and ethylene contents. Genes annotated as $\mathrm{GH} 3$ and auxin responsive protein were detected in 'Huangbao' and 'Guanxi', all of them were down regulated in 'Huangbao'. It has been reported that $A B C$ transporter B family member 19 (ABCB19) is capable of transporting auxin in plant [59]. In this study, $A B C B 19$ wasonly detected in 'Guanxi'. Taken together, although $G H 3$ was highly expressed in the pericarp of 'Guanxi' ripe fruits, the carotenoid contents were significantly lower in 'Guanxi' than that in 'Huangbao'. However, low expression levels of genes encoding for Auxin responsive protein and ABCB19 were associated with high carotenoid contents in 'Huangbao'. Auxin responsive protein and ABCB19 seem to act as negative regulators of carotenoid accumulation in fruits.

\section{Transcription factors}

The two opposite roles of DELLA protein in regulating GA metabolic pathway were observed in different plant species. In pea (Pisum sativum L.), DELLA protein down regulated the expression of PSGA2ox1 (a GA deactivation gene) [60]. The loss-of-function of DELLA protein induced dwarf phenotype in tomato was demonstrated by CRISPR/Cas9 approach [61]. On the contrary, a functionless SLN1 (a DELLA protein) protein could activate GA signaling pathway in the barley aleurone [62]. In this study, GA2OX and DELLA protein genes were specially expressed in the pericarp of ripe fruits from 'Huangbao' and 'Guanxi', respectively, which indicated that the GA activity might be inhibited in 'Huangbao'. Due to the effect of GA inhibition on carotenoid accumulation, GA deactivation should promote pericarp colouration in 'Huangbao'. It is still unclear whether DELLA protein gene is involved in regulating carotenoid accumulation.

NAC family genes in association with fruit ripening. In banana (Musa paradisiaca), MaNAC1/2 could physically interacte with MaEIL5, a downstream component of ethylene signaling pathway [63]. It has been found that the SINAC1 gene negatively regulated the carotenoid and ethylene biosyntheses in 
tomato fruits (Solanum lycopersium). Overexpression of this gene was accompanied with decrease in transcript levels of SIPSY1, SIACO1 and SIACS2 and increase in expression levels of SILCYb, SILCYe and SICYCB. which consequently decreased the total carotenoid and ethylene contents in fruits [64]. The influences of SINAC1 in carotenoid and ethylene metabolisms could be further demonstrated by antisence suppression of it, as a consequence, lycopene and ethylene contents were much higher in the transgenetic fruits than that in the wild type fruits [65]. Another member of NAC family, SINAC4 acted as a positive regulator in upstream of the ripening regulator RIN [66]. When SINAC4 was silenced by virusinduced gene silencing, fruit ripening was delayed and ethylene production and lycopene accumulation were reduced in tomato fruits [67]. The previous research reveals that NAC family genes play different roles in regulating ethylene and carotenoid metabolic pathways. Four genes encoding for NAC domaincontaining protein were indentified in this study, while three of them were up regulated in 'Huangbao', the last one were down regulated. Further assays need to be carried out to define clear roles of those NAC family genes in modulating carotenoid accumulation in pummelo fruits.

Just like NAC family genes, the members of MADS-box gene family act as a positive regulator or a repressor in regulating carotenoid and ethylene metabolisms. Ripening inhibitor $(R I M)$ is a MADS-box transcription factor. It is involved in regulating carotenoid and ethylene metabolisms in both climacteric and non-climacteric fruits $[68,69]$. MADS-box proteins encoded by SIMBP15, FUL 1 and FUL2 can interact with RIN, when those three genes were silenced individually, the carotenoid and ethylene contents as well as the expression levels of ethylene biosynthetic genes decreased in tomato fruits [70, 71]. CsMADS6 directly bound to the promoters of $P S Y, P D S, \angle C Y B 1$ and $C C D 1$ and trans-activated their expressions, finally, caused the increase in carotenoid content in sweet orange calli [72]. On the other hand, some other members of MADS-box gene family play a reverse role in modulating carotenoid and ethylene accumulation. For example, when SIMBP8 or SIMADS1 was silenced, genes linked to ethylene $(A C O, A C S$, $E R F 1, E 4$ and E8) and carotenoid pathway ( $P S Y 1, P D S$ and $Z D S$ ) were up regulated, which was accompanied by improvement in carotenoid and ethylene biosyntheses [73, 74]. Three MADS-box transcription factors were identified in this study, and all of them were specially expressed in 'Guanxi'.

WRKY transcription factor family is involved in response to stresses in plants [75]. PqWRKY1 acted as a positive regulator in triterpene ginsenoside biosynthetic metabolism in Panax quinquefolius and improved insensitiveness of transgenic Arabidopsis plants to abiotic stesses [76]. It was found that OfWRKY 3 could bind to the promoter region of carotenoid cleavage dioxygenase 4 (OfCCD4) to transcriptionally activate its expression in sweet osmanthus (Osmanthus fragrans) [77]. CCD4 catalyzes the cleavage of carotenoid substrates [78]. When InCCD4 was silenced by CRISPR/Cas 9 system, the total carotenoid content in petals of Ipomoea nil was increased 20-fold in comparison with non-transgenic plants [79]. Those previous reports suggest that WRKY transcription factor seems to facilitate isoprene consumption in terpene biosynthesis and accelerate carotenoid degradation, which lead to weakened accumulation of carotenoids. The present study showed that WRKY was significantly down regulated in 'Huangbao' compared with 'Guanxi'. 
MYB transcription factor family contains a large number of members. For instance, a total of 1986 MYB and MYB-related putative proteins were identified in Gossypium hirsutum genome [80]. Some of MYB members participate in regulating anthocyanin and flavonoid metabolisms [81, 82], while some others are involoved in modulating carotenoid metabolism. Reduced expression of Carotenoid Pigmentation 1 (an R2R3-MYB transcription factor) was associated with improved carotenoid biosynthesis in Mimulus lewisii [83]. Another R2R3-MYB transcriptional factor (CrMYB68) in a stay-green mutant of Citrus reticulata cv Suavissima directly and negatively regulated $\mathrm{CrBCH} 2$ and 9-cis-epoxycarotenoid dioxygenase 5 , as a consequence, the transformation of $a$-carotene and $\beta$-cartoene and the ABA biosynthesis were delayed [84]. In this study, one MYB44 transcription factor and two MYB related proteins were distinctly down regulated in 'Huangbao' in comparison with 'Guanxi'. It has been demonstrated that MYB44 mainly participates in stress response [85], but there isn't any information confirms that it is also involved in regulating carotenoid metabolism. Therefore, it remains unclear whether the three MYB related genes identified in this study are associated with colour development in fruit pericarp.

Genes associated with post translational modification

SIHDT3 is a histone deacetylase and belongs to tomato HD2 family, when SIHDT3 was silenced by RNAi technology, the amount of ethylene production and carotenoid accumulation decreased [86]. SIHDA3, a member of tomato RPD3/HDA1 family of histone deacetylases, played a negative role in regulating carotenoid accumulation in tomato fruits [87]. It can be ovbserved that members from different histone deacetylase families may paly contradictory roles in modulating carotenoid metabolic pathway. In this study, expression level of the gene encoding for histone acetyltransferase was 36.95-fold higher in 'Huangbao' than that of in 'Guanxi'. The present study results likely support the idea that histone acetylation promotes carotenoid accumulation in fruits.

Ethylene-overproducer1 (ETO1)/ETO1-like (EOLs) are components of a CULLIN-3 E3 ubiquitin ligase. EOLs can bind to ACS protein and degrade it, however, this effect can be reversed by 14-3-3 protein which interacts with EOLs and destabilizes them. 14-3-3 protein can also directly bind to ACS protein and maintain stabilization of ACS [88]. Transcript levels of E2 ubiquitin-conjugating enzyme genes were lower in yellow areas compared with orange areas in tomato fruits (Solanum lycopersicum cv Ailsa Craig) [70]. E3 ubiquitin ligase and E2 ubiquitin-conjugating enzyme both are involved in regulating fruit ripening. In this study, in 'Huangbao' pummelo, one 14-3-3-like protein gene was down regulated, additionally, one of the two genes annotated as E2 ubiquitin-conjugating enzyme was up regulated and another one was down regulated. The up regulated E2 ubiquitin-conjugating enzyme gene showed high transcript level in both of the two pummelo cultivars when compared to the down regulated one. Collectively, the up regulated gene encoding for E2 ubiquitin-conjugating enzyme may act as a positive regulator in modulating carotenoid metabolism.

Other metabolisms may provide a metabolic background for carotenoid accumulation

Expression levels of DEGs associated with fatty acid metabolism and lipid transport were significantly lower in 'Huangbao' than that of in 'Guanxi'. It has also been investigated that the some fatty acid 
biosynthetic genes were down regulated in fruits from orange-pericarp mutant in pummelo (Citrus grandis (L.) Osbeck) [89]. Transcript abundance of genes annotated as sugar, water and metal element transporters showed significant changes. Some significantly up regulated genes encoding for ribosomal proteins, mRNA elongation factors and cytochrome c oxidase were observed in 'Huangbao', which suggested that the total metabolic activity was higher in the pericarp of 'Huangbao' ripe fruits compared with 'Guanxi'. Those DEGs involved in other metabolic pathways might provide a metabolic background for lycopene accumulation in the pericarp of ripe fruits from 'Huangbao'.

\section{Conclusions}

Large amount of lycopene accumulated in the pericarp of ripe fruits from 'Huangbao' pummelo was associated with significantly higher expression levels of genes encoding for fructose-bisphosphate aldolase, enolase and pyruvate kinase isozyme, however, transcript levels of genes encoding for phytoene synthase and prolycopene isomerase, those are two key enzymes in lycopene biosynthesis pathway, were significantly lower in 'Huangbao' than that of in 'Guanxi'. Fructose-bisphosphate aldolase, enolase and pyruvate kinase isozyme catalyze the formation of 3-phosphate-glyceraldehyde and pyruvic acid. The two productions are precursors for IPP and DMAPP biosyntheses in MEP pathway, subsequently, IPP and DMAPP act as precursors for carotenoid biosynthesis. Those results indicate that the regulation in carotenoid metabolism is expanded to the carbon skeleton biosynthetic pathway in the pericarp of pummelo fruits. Further experiments need to be carried out to verify whether the activities of phytoene synthase and prolycopene isomerase are regulated by post-translational modification. Gibberellin-2- $\beta-$ dioxygenase gene was up regulated in 'Huangbao', however, genes related to auxin response and auxin transport, as well as several negative regulators in ethylene signaling pathway were down regulated in 'Huangbao'. It has been reported that phytohormone is involved in regulating carotenoid metabolism. Therefore, the DEGs related to phytohormone metabolism or its singal transduction may participate in modulating carotenoid accumulation in fruits. Additionally, transcription factors (DELLA, NAC, MADS-box, WRKY) and genes encoding for post translational modification proteins (histone acetyltransferase and E2 ubiquitin-conjugating enzyme) involved in regulating ethylene and carotenoid metabolisms were identified in this study. Those findings provide a promising new research direction for better understanding regulation of carotenoid accumulation in fruits.

\section{Methods}

Fruit samples

'Huangbao' was selected from bud mutation of 'Guanxi' pummelo (Citrus grandis (L.) Osbeck) for its red colour pericarp. This phenotype is observed during fruit ripening stage, which is distinctly different with the yellow pericarp of wild type fruits (Fig. 6). 'Huangbao' and 'Guanxi' pummelo cultivars were both grafted on 'Sour pummelo' (Citrus grandis) and planted at $3.0 \mathrm{~m} \times 3.5 \mathrm{~m}$ in a commercial orchard located in Zhangzhou City, China. Ripe fruit samples of each cultivar were harvested at the outside canopy of three 4-year-old trees with same growth vigor. Three fruits were sampled from each tree and each tree 
was taken as one biological replicate. Pericarp of three fruits harvested from each tree was cut into small pieces, mixed together, immediately frozen in liquid nitrogen and then stored at $-80^{\circ} \mathrm{C}$ for RNA isolation and carotenoid measurement.

RNA preparation, cDNA library construction and RNA sequencing

Totoal RNA was extracted with E.Z.N.A. Plant RNA Kit (R6827-02) (OMEGA, Georgia, USA) following the manufacturer's instructions. The purity of RNA extraction was checked with a NanoPhotometer ${ }^{\circledR}$ spectrophotometer (IMPLEN, CA, USA). The concentration of total RNA was measured using Qubit ${ }^{\circledR}$ RNA Assay Kit in Qubit ${ }^{\circledR} 2.0$ flurometer (Life Technologies, CA, USA). The RNA integrity number (RIN) was evaluated with the Agilent 2100 bioanalyzer (Agilent Technologies, CA, USA) using the RNA Nano 6000 Assay Kit. $A_{260} / A_{280}$ ratio more than $1.8, A_{260} / A_{230}$ ratio up to 2.0 and $R I N$ exceeded 8.0 were considered as the criteria for the high quality RNA samples.

A total amount of $3 \mu \mathrm{g}$ RNA per sample was used as input material for the RNA sample preparations. Sequencing libraries were generated using NEBNext ${ }^{\circledR} U$ Utra $^{T M}$ RNA Library Prep Kit for Illumina ${ }^{\circledR}$ (NEB, USA) following manufacturer's recommendations and index codes were added to attribute sequences to each sample. Briefly, mRNA was enriched from total RNA using poly-T oligo-attached magnetic beads and fragmented using divalent cations under elevated temperature in NEBNext First Strand Synthesis Reaction Buffer (5X). The cleaved mRNA fragments were primerd with random hexamers and reverse transcribed into single-stranded cDNA using M-MuLV reverse transcriptase (RNase $\mathrm{H}-$ ). In the next step, the second-stand cDNA synthesis was synthesized using DNA polymerase I and RNase H. Remaining overhangs were converted into blunt ends via exonuclease/polymerase activities. After adenylation of $3^{\prime}$ ends of DNA fragments, NEBNext Adaptor with hairpin loop structure were ligated to prepare for hybridization. cDNA fragments with lengths between 150-200bp were preferentially selected using AMPure XP system (Beckman Coulter, Beverly, USA). Then $3 \mu$ L USER Enzyme (NEB, USA) was used with size-selected, adaptor-ligated cDNA at $37^{\circ} \mathrm{C}$ for 15 min followed by 5 min at $95^{\circ} \mathrm{C}$ before PCR. Subsequently, the selected cDNAs were amplified by PCR using phusion high-fidelity DNA polymerase, universal PCR primers and index $(X)$ primer. Finally, cDNA libraries were purified with AMPure XP system and quantified using the Agilent 2100 bioanalyzer. The cDNA libraries were sequenced on an Illumima Hiseq 4000 platform.

Sequence quality control and ungiene assembly

Raw data (raw reads) of fastq format were firstly processed through in-house perl scripts. In this step, low quality reads and reads containing adapter or ploy- $\mathrm{N}$ were removed, and the remaining reads were taken as clean reads. Q30 and GC-content of the clean data were calculated. All the following analyses were based on clean reads with high quality. The clean reads were deposited in the National Center for Biotechnology Information (NCBI) short Read Archive database (https://www.ncbi.nlm.nih.gov/sra) with accession number of SRP131839. All clean reads were used for de novo assembly using Trinity software 
(https://github.com/trinityrnaseq/trinityrnaseq/wiki) with min-kmer-cov set to 2 by default and all other parameters set default.

Functional annotation of unigenes

Assembled unigenes were annotated using BLAST (http://blast.ncbi.nlm.nih.gov/Blast.cgi) alignment with an $E$ value threshold of $1 e^{-5}$ against the following opening biological information databases: NCBI non-redundant protein sequences (NR) (ftp://ftp.ncbi.nih.gov/blast/db/), a manually annotated and reviewed protein sequence database (Swiss-Prot) (http://www.uniprot.org/), Clusters of Orthologous Groups (COG) (http://www.ncbi.nlm.nih.gov/COG/), euKaryotic Orthologous Groups (KOG) (http://www.ncbi.nlm.nih.gov/KOG/), Evolutionary Genealogy of Genes: non-supervised orthologous groups (eggNOG) (http://eggnogdb.embl.de/), gene ontology (GO) (http://www.geneontology.org/) and Kyoto Encyclopedia of Genes and Genomes (KEGG) (http://www.genome.jp/kegg/). KEGG pathway analysis was conducted using KOBAS2.0 (http://kobas.cbi.pku.edu.cn/help.do) for KEGG Orthology assignments. Putative amino acid sequences of unigenes were blasted against the Protein family database (Pfam) (http://pfam.xfam.org/) using HMMER software (http://hmmer.org/) with the e-value cutoff of $1 \mathrm{e}^{-10}$ to obtain the annotated information of unigenes.

Differentially expression analysis of unigenes

Clean data were mapped back onto the assembled transcriptome, and then the read count for each unigene was obtained from the mapping results. The transcript abundance of unigenes was statistically calculated using fragments per kilobase of transcript per million mapped reads (FPKM). Differentially expressed unigenes were identified using the DESeq $R$ package (1.10.1). The resulting $P$ values were adjusted using the Benjamini and Hochberg's approach for controlling the false discovery rate (FDR). FDR threshold $<0.01$ and fold change $\geq 2$ were used to measure the significance of the unigene expression differences.

GO and KEGG pathway enrichment analysis

GO enrichment analysis of DEGs was implemented by the topGO R packages (http://www.bioconductor.org/packages/release/bioc/html/topGO.html) based Kolmogorov-Smirnov test. KO-Based Annotation System (KOBAS) software (http://kobas.cbi.pku.edu.cn/help.do) was used to test the statistical enrichment of DEGs in KEGG pathways. Corrected P-value $\leq 0.05$ was defined as significantly enriched in DEGs.

Carotenoid measurements

Carotenoid extraction The procedure of carotenoid isolation was based on the method reported by Li etal. [90] with some modifications. Briefly, carotenoids were extracted from $1.2 \mathrm{~g}$ of pericarp powder with $6 \mathrm{~mL}$ of $\mathrm{n}$-hexane: acetone: ethanol $(2: 1: 1, \mathrm{v} / \mathrm{v} / \mathrm{v})$ solution at $4{ }^{\circ} \mathrm{C}$ for $20 \mathrm{~h}$ under dim light. The solution mixture was centrifuged at $12000 \mathrm{~g}$ and $4{ }^{\circ} \mathrm{C}$ for $10 \mathrm{~min}$. The supernatant was transferred to $20 \mathrm{~mL}$ glass tube. 
The remaining pellet was suspended in $6 \mathrm{~mL}$ of extraction solution and maintained at $4{ }^{\circ} \mathrm{C}$ for $4 \mathrm{~h}$. Subsequently, the supernatants were combined and blown to dryness using $\mathrm{N}_{2}$. The dried carotenoid extracts were dissolved in $1 \mathrm{~mL}$ of methyl tert-butyl ether (MTBE) contaning $0.1 \%(\mathrm{w} / \mathrm{v})$ butylated hydroxytoluene (BHT) and filtered through $0.22 \mathrm{~mm}$ nylon membrane. This filtered solution was used for detecting lycopene and $\beta$-carotene contents. Used the same carotenoid extraction method, when the dried carotenoid extracts were dissolved in $1 \mathrm{~mL}$ ethyl acetate containing $0.1 \%(\mathrm{w} / \mathrm{v}) \mathrm{BHT}$, the filtered solution was used to measure lutein, zeaxanthin and $\beta$-cryptoxanthin contents.

Carotenoid quantification High performance liquid chromatography (HPLC) analyses of carotenoid extracts were performed on a Waters e2695 (Waters, Massachusetts, USA) unit equipped with a photodiode array detector (PAD). Lycopene and $\beta$-carotene was separated on an AcclaimTM $\mathrm{C}_{30}$ column $(4.6 \times 250 \mathrm{~mm}, 5 \mu \mathrm{M})$, the column temperature was maintained at $35^{\circ} \mathrm{C}$, with a flow rate of $1.0 \mathrm{~mL} \mathrm{~min}{ }^{-1}$ and a detection wavelength of $450 \mathrm{~nm} .10 \mu \mathrm{L}$ of samples was injected with auto sampler. Carotenoids was gradient eluted with methanol/acetonitrile $(1: 3, \mathrm{v} / \mathrm{v}$; eluent $\mathrm{A})$ containing $0.1 \%(\mathrm{w} / \mathrm{v}) \mathrm{BHT}$ and MTBE (eluent B) containing $0.1 \%(\mathrm{w} / \mathrm{v}) \mathrm{BHT}$. The gradient elution procedure was as follows: $90 \% \mathrm{~A}$ and $10 \% \mathrm{~B}$ initially, from $90-70 \% A$ in 4 min, from $70-30 \% A$ in 4 min, from $30-70 \% A$ in 6 min, from $70-90 \% A$ in 2 min, and isocratic at $90 \% \mathrm{~A}$ and $10 \% \mathrm{~B}$ for $10 \mathrm{~min}$. Lutein, zeaxanthin and $\beta$-cryptoxanthin were measured with another strategy. This three carotenoid components were separated on a $\mathrm{YMC}, \mathrm{C}_{30}$ caroteniod column, $4.6 \times 250 \mathrm{~mm}, 5 \mu \mathrm{m}$ (YMC, Wilmington, NC), the column temperature was maintained at $25^{\circ} \mathrm{C}$, with a flow rate of $1.0 \mathrm{~mL} \mathrm{~min}^{-1}$ and a detection wavelength of $450 \mathrm{~nm} .5 \mu \mathrm{L}$ of samples was injected with auto sampler. Solvent A consisted of methanol/water $(97: 3, \mathrm{v} / \mathrm{v})$, and solvent B consisted of $100 \% \mathrm{MTBE}$, both eluents contained $0.1 \%(\mathrm{w} / \mathrm{v}) \mathrm{BHT}$. Carotenoids were gradient eluted according to the following procedure: Omin, $90 \% \mathrm{~A}$ and $10 \% \mathrm{~B} ; 4 \mathrm{~min}, 70 \% \mathrm{~A}$ and $30 \% \mathrm{~B} ; 6 \mathrm{~min}, 60 \% \mathrm{~A}$ and $40 \% \mathrm{~B} ; 8 \mathrm{~min}, 30 \% \mathrm{~A}$ and $70 \% \mathrm{~B} ; 12 \mathrm{~min}, 70 \% \mathrm{~A}$ and $30 \% \mathrm{~B} ; 14 \mathrm{~min}, 90 \% \mathrm{~A}$ and $10 \% \mathrm{~B}$ and $24 \mathrm{~min}, 90 \% \mathrm{~A}$ and $10 \% \mathrm{~B}$. Lycopene, $\beta-$ carotene, lutein, zeaxanthin and $\beta$-cryptoxanthin standards were purchased from Yuanye company (Yuanye, Shanghai, China). Carotenoid components were quantified by both the retention times and absorption spectrum curves.

qRT-PCR validation

A CTAB protocol was used to extract total RNA from pericarp according to Zhu etal. [91]. $4 \mu \mathrm{g}$ of total RNA was used for first stand cDNA synthesis by PrimeScript RT reagent Kit with gDNA Eraser (Perfect Real Time RR047A) (Takara, Dalian, China) following manufacturer's instructions. The tenfold diluted cDNA was used as template for qRT-PCR. Determination of the relative expression levels was performed on a Mastercycler ep realplex instrument (Eppendorf, Hamburg, Germany), using the SYBR Premix DimerEraser Kit (Perfect Real Time RR091A) (Takara, Dalian, China). The assay mixture was in a final volume of $25 \mu \mathrm{L}$, which consisted of $12.5 \mu \mathrm{L}$ of SYBR Premix DimerEraser $(2 \times), 1 \mu \mathrm{L}$ of each primer (10 $\mu \mathrm{M}), 1 \mu \mathrm{L}$ of cDNA template, and $9.5 \mu \mathrm{L}$ of RNase-free water. Cycling protocol consisted of an initial step of $95^{\circ} \mathrm{C}$ for $30 \mathrm{~s}$, then followed by 40 cycles of $95^{\circ} \mathrm{C}$ for $5 \mathrm{~s}, 55^{\circ} \mathrm{C}$ for $30 \mathrm{~s}$ and $72{ }^{\circ} \mathrm{C}$ for $30 \mathrm{~s}$, and completed with a melting curve analysis. The specificity of qRT-PCR primers was confirmed by melting 
curve. The primer sequences used in this study were designed using primer3 (http://bioinfo.ut.ee/primer30.4.0/primer3) and listed in Additional file 9. The fold change of gene expression was calculated by using the formula $2^{-\triangle \Delta C T}$ and normalized to the actin gene of Citrus maxima (GenBank accession number: GQ389668.1).

\section{Abbreviations}

IPP: Isopentenylpyrophosphate; GA: Gibberenllin; DMAPP: Dimethylallyl pyrophosphate; MEP:

Methylerythritol phosphate; GGPP: geranylgeranyl diphosphate; PSY: Phytoene synthase; PDS: Phytoene desaturase; ZDS: Z-carotene desaturase; CRTISO: Carotenoid isomerase; $\beta$-LCY: Lycopene $\beta$-cyclase; $\varepsilon$ $\mathrm{LCY}$ : Lycopene $\varepsilon$-cyclase; $\mathrm{BCH}$ : $\beta$-ring carotene hydroxylase; $\mathrm{E} \mathrm{CH}$ : $\varepsilon$-ring carotene hydroxylase; $\mathrm{DEG}$ : Differentially expressed gene; BP: Biological process; MF: Molecular founction; CC: cellular component; FPKM: Fragments per kilobase of transcript per million mapped reads; GA2OX: Gibberellin-2-betadioxygenase; ACS: 1-aminocyclopropane-1-carboxylate synthase; ACO: 1-aminocyclopropane-1carboxylate oxidase; GH3: Indole-3-acetic acid-amido synthetase; EBF: EIN3-binding F-box protein; EIN3: Ethylene insensitive 3; ABC: ATP binding cassette; HPLC: High performance liquid chromatography; qRTPCR: quantitative real time PCR; GRP: Glycine-rich RNA-binding protein; CKX: cytokinin dehydrogenase; ACC: 1-aminocyclopropane-1-carboxylate; EIL: Ethylene insensitive-like proteins; IAA: Indole-3-acetic acid; CCD4: carotenoid cleavage dioxygenase 4; EOLs: Ethylene-overproducer1 (ET01)/ET01-like; MTBE: Methyl tert-butyl ether; BHT: Butylated hydroxytoluene; HPLC: High performance liquid chromatography

\section{Declarations}

Acknowledgements

None.

Funding

This work was supported by Fujian Special Fund in The Public Interest (Grant No. 2018R1013-12 and 2017R1013-3) and National Science and Technology Infrastructure Platform granted by Ministry of Science and Technology and Ministry of Finance of People's Republic of China (Grant No. NICGR2018069). The funders had no role in study design, data collection and analysis and writing of the manuscript.

Availability of data and materials

The clean reads are available in the NCBI short Read Archive repository (https://www.ncbi.nlm.nih.gov/sra), available from accession SRP131839. All supporting data were included within the manuscript and its additional fiels. Xinkun Lu obtained the permission of fruit sample collection from the orchard owner and undertook the formal identification of the samples and provide details of any voucher specimens deposited. 
Authors' contributions

XL conceived and designed the experiment. XL and YL (Yanjin Lin) collected plant samples. YL (Yanqing Lu) analyzed the data, carried out the qRT-PCR and the carotenoid measurement experiments and drafted the manuscript. All authors read and approved the final manuscript.

Competing interests

The authors declare that they have no competing interests.

Consent for publication

Not applicable.

Ethics approval and consent to participate

Not applicable.

Author details

Institute of Pomology, Fujian Academy of Agricultural Sciences, Fuzhou 350013, People's Republic of China

\section{References}

1. Phillip D, Young AJ. Occurrence of the carotenoid lactucaxanthin in higher plant LHC II. Photosynth Res. 1995;43:273- 282.

2. Sandmann G, Kuhn M, Böger P. Carotenoids in photosynthesis: Protection of D1 degradation in the light. Photosynth Res . 1993;35:185- 190.

3. Alboresi A, Dall'Osto L, Aprile A, Carillo P, Roncaglia E, Cattivelli L, Bassi R. Reactive oxygen species and transcript analysis upon excess light treatment in wild-type Arabidopsis thaliana vs a photosensitive mutant lacking zeaxanthin and lutein. BMC Plant Biol. 2011;11:62.

4. Prasad KN, Chew LY, Khoo HE, Yang B, Azlan A, Ismail A. Carotenoids and antioxidant capacities from CanariumodontophyllumMiq. fruit. Food Chem. 2011;124(4): 1549-1555.

5. Sponsel VM. The deoxyxylulose phosphate pathway for the biosynthesis of plastidic isoprenoids: early days in our understanding of the early stages of gibberellin biosynthesis. J plant growth regul.

2002;20:332-345.

6. Song MH, Lim SH, Kim JK, Jung ES, John KMM, You MK, Ahn SN, Lee CH, Ha SH. In planta cleavage of carotenoids by Arabidopsis carotenoid cleavage dioxygenase 4 in transgenic rice plants. Plant Biotechnol Rep. 2016;10:291-300. 
7. Wang Y, Ding GQ, Gu TT, Ding J, Li Y. Bioinformatic and expression analyses on carotenoid dioxygenase genes in fruit development and abiotic stress responses in Fragaria vesca. Mol Genet Genomics. 2017;292: 895-907.

8. Yamaguchi M. Role of carotenoid $\beta$-cryptoxanthin in bone homeostasis. J Biomed Sci. 2012;19:36.

9. Nisar N, Li L, Lu S, Khin NC, Pogson BJ. Carotenoid metabolism in plants. Mol Plant. 2015;8: 68-82.

10. Schweiggert RM, Steingass CB, Heller A, Esquivel P, Carle R. Characterization of chromoplasts and carotenoids of red- and yellow-fleshed papaya (Carica papaya L.). Planta. 2011;234:1031-1044.

11. Lado J, Rodrigo MJ, Cronje P, Zacarias L. Involvement of lycopene in the induction of tolerance to chilling injury in grapefruit. Posth Biol Technol. 2015;100:176-186.

12. Nogueira M, Mora L, Enfissi EMA, Bramley PM, Fraser PD. Subchromoplast sequestration of carotenoids affects regulatory mechanisms in tomato lines expressing different carotenoid gene combinations. The Plant Cell. 2013;25:4560-4579.

13. Karppinen K, Zoratti L, Sarala M, Carvalho E, Hirsimäki J, Mentula H, Martens S, Häggman H, Jaakola L. Carotenoid metabolism during bilberry (Vaccinium myrtillus L.) fruit development under different light conditions is regulated by biosynthesis and degradation. BMC Plant Biol. 2016;16:95.

14. Lee WL, Huang JZ, Chen LC, Tsai CC, Chen FC. Developmental and LED light source modulation of carotenogenic gene expression in Oncidium gower ramsey flowers Plant Mol Biol Rep. 2013;31:14331445 .

15. Lester GE, Eischen F. Beta-carotene content of postharvest orange-fleshed muskmelon fruit: Effect of cultivar, growing location and fruit size. Plant Foods Hum Nutr. 1996;49:191-197.

16. Taber H, Perkins-Veazie P, Li SS, White W, Rodermel S, Xu Y. Enhancement of tomato fruit lycopene by potassium is cultivar dependent. Hortsci. 2008;43(1):159-165.

17. Erdoğan A, Demirel Z, Eroğlu AE, Dalay MC. Carotenoid Profile in Prochlorococcus sp. and Enrichment of Lutein Using Different Nitrogen Sources. J Appl Phycol . 2016; 28 (6 ): 3251- 3257.

18. Muzolf-Panek M, Kleiber T, Kaczmarek A. Effect of increasing manganese concentration in nutrient solution on the antioxidant activity, vitamin $\mathrm{C}$, lycopene and polyphenol contents of tomato fruit. Food Addit Contam A. 2017;34(3):379-389.

19. Lu Q, Huang XJ, Lv SY, Pan SY. Carotenoid profiling of red navel orange "Cara Cara" harvested from five regions in China. Food Chem. 2017;232:788-798.

20. Montefiori M, Costa G, McGhie T, Ferguson, AR. Effects of light and temperature on colour changes in ripening fruit of Actinidia macrosperma. Acta Horticul. 2005;682(1):185-190 
21. Carmona L, Zacarias L, Rodrigo MJ. Stimulation of coloration and carotenoid biosynthesis during postharvest storage of 'Navelina' orange fruit at 12 degrees C. Posth Biol Technol. 2012;74:108-117.

22. Fanciullino $A L$, Cercos $M$, Dhuique-Mayer $C$, Froelicher $Y$, Talon $M$, Ollitrault $P$, Morillon R. Changes in carotenoid content and biosynthetic gene expression in juice sacs of four orange varieties (Citrus sinensis) differing in flesh fruit color. J Agric Food Chem. 2008;56(10):3628-3638.

23. Wei X, Hu H, Tong HR, Gmitter Jr Frederick G. Profiles of gene family members related to carotenoid accumulation in citrus genus. J. Plant Biol. 2017;60:1-10.

24. Tao J, Zhang SL, Xu JG, Liu CR. Analysis of major carotenoid composition and its content in citrus fruit. Sci Agric Sinica. 2003;36 (10):1202-1208.

25. Ben AS, Rodrigo MJ, Saddoud O, Zacarias L, Hajlaoui MR, Mars M. Carotenoids and colour diversity of traditional and emerging Tunisian orange cultivars (Citrus sinensis (L.) Osbeck). Sci Hortic. 2018;227:296-304.

26. Smita S, Rajwanshi R, Lenka SK, Katiyar A, Chinnusamy V, Bansal KC. Expression profile of genes coding for carotenoid biosynthetic pathway during ripening and their association with accumulation of lycopene in tomato fruits. India Acad Sci. 2013;92(3):363-368.

27. Li PR, Zhang SJ, Zhang SF, Li F, Zhang H, Liu XY, Wu J, Wang XW, Sun RF. Carotenoid identification and molecular analysis of carotenoid isomerase-encoding BrCRTISO, the candidate gene for inner leaf orange coloration in Chinese cabbage. Mol Breeding. 2015;35:72.

28. Yoshioka S, Aida R, Yamamizo C, Shibata M, Ohmiya A. The carotenoid cleavage dioxygenase 4 (CmCCD4a) gene family encodes a key regulator of petal color mutation in chrysanthemum. Euphyt. 2012;184:377-387.

29. Gady ALF, Vriezen WH, Wal MHBJVD, Huang PP, Bovy AG, Visser RGF, Bachem CWB. Induced point mutations in the phytoene synthase 1 gene cause differences in carotenoid content during tomato fruit ripening. Mol Breeding. 2012;29:801-812.

30. Fu XM, Feng C, Wang CY, Yin XR, Lu PJ, Grierson D, Xu CJ, Chen KS. Involvement of multiple phytoene synthase genes in tissueand cultivar-specific accumulation of carotenoids in loquat. J Exp Bot. 2014;65(16): 4679-4689.

31. Li QR, Farre G, Naqvi S, Breitenbach J, Sanahuja G, Bai C, Sandmann G, Capell T, Christou P, Zhu CF. Cloning and functional characterization of the maize carotenoid isomerase and $\beta$-carotene hydroxylase genes and their regulation during endosperm maturation. Transgenic Res. 2010;19:1053-1068.

32. Xu Q, Yu KQ, Zhu AD, Ye JL, Liu Q, Zhang JC, Deng XX. Comparative transcripts profiling reveals new insight into molecular processes regulating lycopene accumulation in a sweet orange (Citrus sinensis) red-flesh mutant. BMC Genomics. 2009;10:540. 
33. Fraser PD, Hedden P, Cooke DT, Bird CR, Schuch W, Bramley PM. The effect of reduced activity of phytoene synthase on isoprenoid levels in tomato pericarp during fruit development and ripening. Planta. 1995.196:321-326.

34. Borthakur PK, Ranjit K. Effect of growth regulators on chlorophyll and carotenoid content in the flavedo of Baramasi lemon fruits during storage. Haryana J Hortic Sci. 2005;34(1/2):42-44.

35. Dijkstra C, Adams E, Bhattacharya A, Page AF, Anthony P, Kourmpetli S, Power JB, Lowe KC, Thomas SG, Hedden P, Phillips AL, Davey MR. Over-expression of a gibberellin 2-oxidase gene from Phaseolus coccineus $\mathrm{L}$. enhances gibberellin inactivation and induces dwarfism in Solanum species. Plant Cell Rep. 2008;27:463-470.

36. Löhr B, Streitner C, Steffen A, Lange T, Staiger D. A glycine-rich RNA-binding protein affects gibberellin biosynthesis in Arabidopsis. Mol Biol Rep. 2014;41:439-445.

37. Tonelli C, Salvi C, Gavazzi G, Marziani LGP, Rossi G, Longo C. Benzyladenine partially restores the normal phenotype in a chlorophyll mutant of maize. J Plant Physiol. 1989;133(6):713-718.

38. Bai BZ, Kastori R. Physiological effects of the cytokinin 6-BA in sunflower. Oil Crops of China. 1990;4: 95-101.

39. Tung MS. Cytokinins-efficiently enhanced pigment production in detached cotyledons of dark-grown cucumber seedlings. J Agric Forest. 1997;46(2):85-91.

40. Zhao J, Bai WQ, Zeng QW, Song SQ, Zhang M, Li XB, Hou L, Xiao YH, Luo M, Li DM, Luo XY, Pei Y. Moderately enhancing cytokinin level by down-regulation of GhCKX expression in cotton concurrently increases fiber and seed yield. Mol Breeding. 2015;35:60.

41. Barry CS, Giovannoni JJ. Ethylene and fruit ripening. J Plant Growth Regul. 2007;26:143-159.

42. Gao L, Zhao WH, Qu HO, Wang QS, Zhao LX. The yellow-fruited tomato 1 (yft1) mutant has altered fruit carotenoid accumulation and reduced ethylene production as a result of a genetic lesion in ETHYLENE INSENSITIVE2. Theor AppI Genet, 2016;129:717-728.

43. Carmona L, Rodrigo MJ, Zacarias L. Exploring the involvement of ethylene in the regulation of color changes in citrus fruit. Acta Hortic. 2012;934:879-885.

44. Alos E, Martinez-Fuentes A, Reig C, Mesejo C, Zacarias L, Agusti M, Rodrigo MJ. Involvement of ethylene in color changes and carotenoid biosynthesis in loquat fruit (Eriobotrya japonicaLindl. cv. Algerie). Posth Biol Technol. 2019;149:129-138.

45. Hou BZ, Li CL, Han YY, Shen YY. Characterization of the hot pepper (Capsicum frutescens) fruit ripening regulated by ethylene and ABA. BMC Plant Biol. 2018;18:162. 
46. Tsuchisaka A, Yu GX, Jin HL, Alonso JM, Ecker JR, Zhang XM, Gao S, Theologis A. A combinatorial interplay among the 1-aminocyclopropane-1-carboxylate isoforms regulates ethylene biosynthesis in Arabidopsis thaliana. Genetics. 2009;183(3):979-1003.

47. Ruduś I, Sasiak M, Kępczyński J. Regulation of ethylene biosynthesis at the level of 1aminocyclopropane-1-carboxylate oxidase (ACO) gene. Acta Physiol Plant. 2013;35:295-307.

48. Xie XL, Shen SL, Yin XR, Xu Q, Sun CD, Grierson D, Ferguson I, Chen KS. Isolation, classification and transcription profiles of the AP2/ERF transcription factor superfamily in citrus. Mol Biol Rep. 2014;41:4261-4271.

49. Lee JM, Joung JG, McQuinn R, Chung MY, Fei ZJ, Tieman D, Klee H, Giovannoni J. Combined transcriptome, genetic diversity and metabolite profiling in tomato fruit reveals that the ethylene response factor SIERF6 plays an important role in ripening and carotenoid accumulation. Plant J. 2012;70(2):191204.

50. Chung MY, Vrebalov J, Alba R, Lee JM, McQuinn R, Chung JD, Klein P, Giovannoni J. A tomato ( Solanum lycopersicum) APETALA2/ERF gene, SIAP2a, is a negative regulator of fruit ripening. Plant J. 2010;64(6):936-947.

51. Konishi M, Yanagisawa S. Ethylene signaling in Arabidopsis involves feedback regulation via the elaborate control of EBF2 expression by EIN3. Plant J. 2008;55(5):821-831.

52. Fu CC, Han YC, Kuang JF, Chen JY, Lu WJ. Papaya CpEIN3a and CpNAC2 co-operatively regulate carotenoid biosynthesis-related genes $C p P D S 2 / 4, C p L C Y$-e and $C p C H Y-b$ during fruit ripening. Plant Cell Physiol. 2017;58(12):2155-2165.

53. Wang RH, Yuan XY, Meng LH, Zhu BZ, Zhu HL, Luo YB, Fu DQ. Transcriptome analysis provides a preliminary regulation route of the ethylene signal transduction component, SIEIN2, during tomato ripening. PLOS ONE. 2016;11 (12):e0168287.

54. Zhu ZQ, Guo HW. Genetic basis of ethylene perception and signal transduction in Arabidopsis. J Integr Plant Biol. 2008; 50(7):808-815.

55. Deng H, Pirrello J, Chen Y, Li N, Zhu SH, Chirinos X, Mondher B, Liu YS, Liu MC. A novel tomato F-box protein, SIEBF3, is involved in tuning ethylene signaling during plant development and climacteric fruit ripening. The Plant J. 2018;95(4): 648-658.

56. Su LY, Diretto G, Purgatto E, Danoun S, Zouine M, Li ZG, Roustan JP, Bouzayen M, Giuliano G, Chervin C. Carotenoid accumulation during tomato fruit ripening is modulated by the auxin-ethylene balance. BMC Plant Biol. 2015;15:114.

57. Ludwig-Muller J, Julke S, Bierfreund NM, Decker EL, Reski R. Moss (Physcomitrella patens) GH3 proteins act in auxin homeostasis. New Phytol. 2009;181(2):323-338. 
58. Thula S, Akash, NandKiran N, Rahul K. A ripening-induced $S / G H 3-2$ gene regulates fruit ripening via adjusting auxin-ethylene levels in tomato (Solanum lycopersicum L.). Plant Mol Biol. 2018;98(4/5):455469 .

59. Cho, MS, Henry, EM, Lewis, DR, Wu, GS, Muday, GK, Spalding, EP. Block of ATP-binding cassette B19 ion channel activity by 5-nitro-2-(3-phenylpropylamino)-benzoic acid impairs polar auxin transport and root gravitropism. Plant Physiol. 2014;166(4):2091-2099.

60. O'Neill DP, Davidson SE, Clarke VC, Yamauchi Y, Yamaguchi S, Kamiya Y, Reid JB, Ross JJ. Regulation of the gibberellin pathway by auxin and DELLA proteins. Planta. 2010;232:1141-1149.

61. Tomlinson L, Yang Y, Emenecker R, Smoker M, Taylor J, Perkins S, Smith J, MacLean D, Olszewski NE, Jones JDG. Using CRISPR/Cas9 genome editing in tomato to create a gibberellin-responsive dominant dwarf DELLA allele. Plant Biotechnol J. 2019;17(1):132-140.

62. Chen KG, Tian SL, Yandell BS, Kaeppler SM, An YC. Loss-of-function of DELLA protein SLN1 activates GA signaling in barley aleurone. Acta Physiol Plant. 2010;32:789-800.

63. Shan W, Kuang JF, Chen L, Xie H, Peng HH, Xiao YY, Li XP, Chen WX, He QG, Chen JY, Lu WJ. Molecular characterization of banana NAC transcription factors and their interactions with ethylene signalling component EIL during fruit ripening. J Exp Bot. 2012;63(14):5171-5187.

64. Ma NN, Feng HL, Meng X, Li D, Yang DY, Wu CG, Meng QW. Overexpression of tomato SINAC1 transcription factor alters fruit pigmentation and softening. BMC Plant Biol. 2014;14:351.

65. Meng C, Yang DY, Ma XC, Zhao WY, Liang XQ, Ma NN, Meng QW. Suppression of tomato sINAC1 transcription factor delays fruit ripening. J Plant Physiol. 2016;193:88-96.

66. Zhu MK, Chen GP, Zhou SA, Tu Y, Wang Y, Dong TT, Hu ZL. A New tomato NAC (NAM/ATAF1/2/CUC2) transcription factor, SINAC4, functions as a positive regulator of fruit ripening and carotenoid accumulation. Plant Cell Physiol. 2014;55(1):119-135.

67. Kou XH, Zhao YN, Wu CE, Jiang BL, Zhang Z, Rathbun JR, He YL, Xue ZH.SNAC4 and SNAC9 transcription factors show contrasting effects on tomato carotenoids biosynthesis and softening. Posth Biol Technol. 2018;144:9-19.

68. Martel C, Vrebalov J, Tafelmeyer P, Giovannoni JJ. The tomato MADS-box transcription factor RIPENING INHIBITOR interacts with promoters involved in numerous ripening processes in a COLORLESS NONRIPENING-dependent manner. Plant Physiol. 2011;157(3):1568-1579.

69. Dong TT, Chen GP, Tian SB, Xie QL, Yin WC, Zhang YJ, Hu ZL. A non-climacteric fruit gene CaMADSRIN regulates fruit ripening and ethylene biosynthesis in climacteric fruit. PLoS ONE. 2014;9(4): e95559. 
70. Wang YY, Wang WH, Cai JH, Zhang YR, Qin GZ, Tian SP. Tomato nuclear proteome reveals the involvement of specific E2 ubiquitin-conjugating enzymes in fruit ripening. Gen Biol. 2014;15:548.

71. Yin WC, Yu XH, Chen GP, Tang BY, Wang YS, Liao CG, Zhang YJ, Hu ZL. Suppression of SIMBP15 inhibits plant vegetative growth and delays fruit ripening in tomato. Front Plant Sci. 2018;9:938.

72. Lu SW, Zhang Y, Zhu KJ, Yang W, Ye JL, Chai LJ, Xu Q, Deng XX. The citrus transcription factor CsMADS6 modulates carotenoid metabolism by directly regulating carotenogenic genes. Plant Physiol. 2018;176(4):2657-2676.

73. Dong TT, Hu ZL, Deng L, Wang Y, Zhu MK, Zhang JL, Chen GP. A tomato MADS-box transcription factor, SIMADS1, acts as a negative regulator of fruit ripening. Plant Physiol. 2013;163(2):1026-1036.

74. Yin WC, Hu ZL, Cui BL, Guo XH, Hu JT, Zhu ZG, Chen GP. Suppression of the MADS-box gene SIMBP8 accelerates fruit ripening of tomato (Solanum lycopersicum). Plant Physiol Biochem. 2017;118:235-244.

75. Xin PF, Gao CS, Cheng CH, Tang Q, Dong ZX, Zhao LN, Zang GG. Identification and characterization of hemp WRKY transcription factors in response to abiotic stresses. Biol Plantarum. 2016;60(3): 489-495.

76. Sun YZ, Niu YY, Xu J, Li Y, Luo HM, Zhu YJ, Liu MZ, Wu Q, Song JY, Sun C, Chen SL. Discovery of WRKY transcription factors through transcriptome analysis and characterization of a novel methyl jasmonateinduciblePqWRKY1 gene from Panax quinquefolius. Plant Cell Tiss Organ Cult. 2013;114:269277.

77. Han YJ,Wu M, Cao LY, Yuan WJ, Dong MF, Wang XH, Chen WC, Shang FD. Characterization of OfWRKY3, a transcription factor that positively regulates the carotenoid cleavage dioxygenase gene OfCCD4 in Osmanthus fragrans. Plant Mol Biol. 2016;91(4/5):485-496.

78. Lashbrooke, JG, Young, PR, Dockrall, SJ, Vasanth K, Vivier MA. Functional characterisation of three members of the Vitis vinifera L. carotenoid cleavage dioxygenase gene family. BMC Plant Biol. 2013;13:156.

79. Watanabe K, Oda-Yamamizo C, Sage-Ono K, Ohmiya A, Ono M. Alteration of flower colour in Ipomoea nil through CRISPR/Cas9-mediated mutagenesis of carotenoid cleavage dioxygenase 4. Transgenic Res. 2018;27:25-38.

80. Salih H, Gong WF, He SP, Sun GF, Sun JL, Du XM. Genome-wide characterization and expression analysis of MYB transcription factors in Gossypium hirsutum. BMC Genetics. 2016;17:129.

81. Ballester AR, Molthoff J,Vos RD, Hekkert BTL,Orzaez D, Fernandez-Moreno JP, Tripodi P, Grandillo S, Martin C, Heldens J, Ykema M, Granell A, Bovy A. Biochemical and molecular analysis of pink tomatoes: deregulated expression of the gene encoding transcription factor SIMYB12 leads to pink tomato fruit color. Plant Physiol. 2010;152(1):71-84. 
82. Feng K, Liu JX, Duan AQ, Li T, Yang QQ, Xu ZS, Xiong AS. AgMYB2 transcription factor is involved in the regulation of anthocyanin biosynthesis in purple celery (Apiumgraveolens L.). Planta. 2018;248(5):1249-1261.

83. Sagawa JM, Stanley LE, LaFountainAM, Frank HA, Liu C, Yuan YW. An R2R3-MYB transcription factor regulates carotenoid pigmentation in Mimulus lewisii flowers. New Phytol. 2016;209(3):1049-1057.

84. Zhu F, Luo T, Liu CY, Wang Y, Yang HB, Yang W, Zheng L, Xiao X, Zhang MF, Xu RW, Xu JG, Zeng YL, Xu J, Xu Q, Guo WW, Larkin RM, Deng XX, Cheng YJ. An R2R3-MYB transcription factor represses the transformation of alpha - and beta -branch carotenoids by negatively regulating expression of $\mathrm{Cr} B \mathrm{CH} 2$ and CrNCED5 in flavedo of Citrus reticulate. New Phytol. 2017;216(1):178-192.

85. Persak H, Pitzschke A. Dominant repression by Arabidopsis transcription factor MYB44 causes oxidative damage and hypersensitivity to abiotic stress. Int J Mol Sci. 2014;15(2):2517-2537.

86. Guo JE, Hu ZL, Li FF, Zhang LC, Yu XH, Tang BY, Chen GP. Silencing of histone deacetylase SIHDT3 delays fruit ripening and suppresses carotenoid accumulation in tomato. Plant Sci. 2017;265:29-38.

87. Guo JE, Hu ZL, Yu XH, Li AZ, Li FF, Wang YS, Tian SB, Chen GP. A histone deacetylase gene, SIHDA3, acts as a negative regulator of fruit ripening and carotenoid accumulation. Plant Cell Rep. 2018;37: 125135.

88. Yoon G M, Kieber JJ. 14-3-3 regulates 1-aminocyclopropane-1-carboxylate synthase protein turnover in Arabidopsis. Plant Cell. 2013;25(3):1016-1028.

89. Guo F, Yu HW, Xu Q, Deng XX. Transcriptomic analysis of differentially expressed genes in an orangepericarp mutant and wild type in pummelo (Citrus grandis). BMC Plant Biol. 2015;15:44.

90. Li WY, Liu CH, He M, Li JQ, Cai YQ, Ma YH, Xu J. Largely different contents of terpenoids in beef redflesh tangerine and its wild type. BMC Plant Biol. 2017;17: 36.

91. Zhu XQ, Jin XL, Feng YQ, Shen YY. An effective method for isolation of high-quality total RNA from fruit pulps. J Beijing Univ Agric. 2008; 23(2):16-18.

\section{Additional Files And Figures}

Due to technical limitations, tables 1 through 13 are only available as downloads in the supplemental files section.

Additional files

Additional file 1: Figure 1. Functional classification of unigenes annotated in COG databasae. The Y-axis represents the number of annotated unigenes, and the $X$-axis represents the functional classification. (png) Figure 2. Functional classification of unigenes annotated in KOG databasae. Y-axis indicates the number of annotated unigenes, and X-axis indicates the functional classification. (png) Figure 3. 
Functional classification of unigenes annotated in eggNOG databasae. Y-axis shows the number of annotated unigenes, and $\mathrm{X}$-axis shows the functional classification. (png)

Additional file 2: Table 1. Unigenes involved in KEGG enriched pathways. Corrected P-value was calculated by adjusting P-value using bonferroni approach. (xlsx)

Additional file 3: Table 2. Changes of expression levels of all DEGs. (xIsx)

Additional file 4: Figure 4. DEGs belonging to top 10 biological process categories. (PDF)

Additional file 5: Figure 5. DEGs belonging to top 10 cellular component categories. (PDF)

Additional file 6: Figure 6. DEGs belonging to top 10 molecular function categories. (PDF)

Additional file 7: Table 3. Expression levels and functional annotations of other metabolic pathway genes. (xlsx)

Additional file 8: Chromatograms and standard curves obtained from HPLC analysis. (xlsx) Figure 7-8. HPLC chromatograms of carotenoid standards, the peaks were numbered according to the elution sequence. Figure 9-13. Standard curves of carotenoid standards. Figure 14-19. HPLC chromatograms of the carotenoid extracts in the pericarp of ripen fruits from pummelo cv. 'Huangbao'. Figure 20-25. HPLC chromatograms of the carotenoid extracts in the pericarp of ripen fruits from pummelo cv. 'Guanxi'.

Additional file 9: Table 4. Primer and gene sequences used for the validation of expression levels of DEGs with qRT-PCR. (xIsx)

Figure titles

Fig. 1 Distribution of unigene length.

Fig. 2 Distribution of the homology search of unigenes against the $\mathrm{Nr}$ database. (a) E-value distribution of the top Blastx hits for unigenes with a cut-off E-value of $1 e^{-5}$. (b) Species distribution of the top Blastx hits for unigenes with a cut-off E-value of $1 e^{-5}$

Fig. $3 \mathrm{GO}$ classification of unigenes. A total of 32164 unigenes were annotated within $\mathrm{GO}$ database into three main categories: biological process, cellular component and molecular function. $Y$ - axis indicates the number of unigenes while $\mathrm{X}$-axis indicates the main $\mathrm{GO}$ categories

Fig. 4 Cluster analysis of DEG expression based on Log2 (FPKM). T13, T14 and T15colums represent three biological replicates of 'Huangbao' pummelo and T16, T17 and T18 columns represent three biological replicates of 'Guanxi' pummelo. Each row represents a unigene. Red, green and black indicate up regulated, down regulated and unchanged, respectively 
Fig. 5 Verification of RNA-sequencing data by qRT-PCR. Relative expression was obtained from qRT-PCR, while FPKM was determinded by RNA-sequencing method. Error bars indicate standard error from three biological replicates

Fig. 6 Ripe fruit of 'Huangbao' (a) and 'Guanxi' (b) cultivars.

\section{Supplementary Files}

This is a list of supplementary files associated with this preprint. Click to download.

- Additionalfile8.xlsx

- Additionalfile2.xIsx

- Additionalfile6.pdf

- Additionalfile1.xlsx

- Additionalfile3.xIsx

- Additionalfile9.xlsx

- Additionalfile4.pdf

- Additionalfile7.xlsx

- Fig3.pdf

- Fig4.pdf

- Fig2.pdf

- Fig1.pdf

- Table4.jpg

- Table11.jpg

- Table5.jpg

- Table3.jpg

- Table2.jpg

- Table1.jpg

- Fig6.pdf

- Fig5.pdf

- Table10.jpg

- Table8.jpg

- Table6.jpg

- Table7.jpg

- Table9.jpg

- Table13.jpg 
- Table12.jpg

- Additionalfile5.pdf

Page $30 / 30$ 